\title{
Experimental Evaluation of SDR-based Equalize and Forward Cooperative Relaying
}

\author{
Ahmed Prince \\ Communication Department \\ Technical Research Center \\ Cairo, Egypt
}

\author{
Mohammed Abdrabou \\ Communication Department \\ Technical Research Center \\ Cairo, Egypt
}

\begin{abstract}
There is a growing interest in Cooperative relaying due to its efficiency to enhance the spatial diversity and the service coverage of communication systems. However, there are various essential challenges that face existent relaying techniques, such as the accumulation of multipath fading effect of the Amplify and Forward (AF) technique and the high computational processing of the Selective Decode and Forward (SDF) relaying techniques. To handle these issues, an Equalize and Forward (EF) relaying technique is proposed to improve the reliability of re-transmission while retaining low computational processing at the relay terminal. In this work, we propose a relaying technique where the relay terminal equalizes the source-relay channel to mitigate the accumulation of the channel without the need of high computational processing and data regeneration. This work presents a practical implementation of cooperative communication framework based on Software Defined Radio (SDR) using GNU Radio, NI USRP 2920 devices, and Single Board Computer (SBC) "ODROID XU4". Two different modulations schemes, Binary Phase Shift Keying (BPSK) and Quadrature Phase Shift Keying (QPSK), are used. The relay terminal utilizes three different cooperative relaying techniques, $\mathrm{AF}$, SDF, and EF. Equal Ratio Combining (ERC) technique is utilized at the intended destination terminal. Performance analysis of $\mathrm{EF}$ relaying technique is evaluated in comparison with the AF and SDF relaying techniques in terms of Bit Error Rate (BER). The experimental results show that the EF relaying technique outperforms the $\mathrm{AF}$ relaying technique and can achieve comparable performance as the SDF relaying technique.
\end{abstract}

Keywords:- Cooperative relaying, SDR, USRP, GNU Radio, $A F, S D F, E F$

\section{INTRODUCTION}

The 5G communication systems are challenged to provide huge number of services through connecting systems in Internet of Things (IoT). The networks which provide these services are comprised of many systems that are characterized by low cost and low power consumption. These systems are designed to cooperate to transmit the data to the intended terminal. Cooperative relaying is now strongly preferred because of its ability to offer high exchange rate, reliability improvement and range extension. Moreover, it is able to overcome many channel issues like multi-path fading and to offer spatial diversity. It can be considered as a substitute to multiple antenna structures when the equipments are restricted to only one antenna (due to the limitations on the equipment size, and/or the extra antennas cost). In cooperative communication schemes, wireless communication started from a source terminal is caught by other terminals (called relay transceiver terminals). The relay terminals process this signal then send it to the intended destination terminal, where various signals copies are combined for reliability improvement. Many researchers are using different available platforms to analyze the cooperative communication networks.

In [1], experimental framework for cooperative communication is built Universal Software Radio Peripheral (USRP) [2] and GNU Radio [3]. The relays use DF and SDF relaying technique without any combining technique utilized at destination terminal. The experimental performance analysis for direct communication, DF and SDF relaying is performed in terms of BER.

In [4], Cooperative communication is experimentally evaluated using USRP and GNU Radio software in order to expand the transmission range. The relays use DF relaying technique. Equal gain combining (EGC) technique is utilized at intended destination terminal. The experimental performance of cooperative multi-relay communication over direct transmission is evaluated by the analysis of BER.

In [5], the experimental implementation for cooperative communication is built using USRPs, GNU Radio and single board computer (ODROID XU4). GMSK modulation scheme is used to transmit text message. The relay terminal uses multihop SDF relaying technique. BER analysis is performed in order to evaluate the simulation and experimental performance of cooperative communication over direct transmission.

In [6], a cooperative communication framework using USRP device and LabVIEW is implemented to evaluate the performance of cooperative communication. The relays use AF relaying technique. Maximum ratio combining (MRC) technique is utilized at intended destination terminal. The experimental performance of cooperative communication over direct transmission is evaluated by the analysis of channel capacity

In this work research an equalize and forward (EF) relaying technique is proposed in order to enhance and improve the performance of cooperative communication. The EF relay terminal equalizes the source-relay channel to mitigate the accumulation of channel without the need of high computational processing and data regeneration. Improvement in the BER for the proposed relaying technique is shown in experimental results. Experimental performance analysis of EF relaying technique is evaluated using USRP 
2920 devices, GNU Radio and Single Board Computer "ODROID XU4" in comparison with the AF and SDF relaying techniques in terms of BER. ERC technique using BPSK and QPSK transmissions are developed. The experimental results declare that EF outperforms $\mathrm{AF}$ and can achieve comparable performance as the SDF relaying technique.

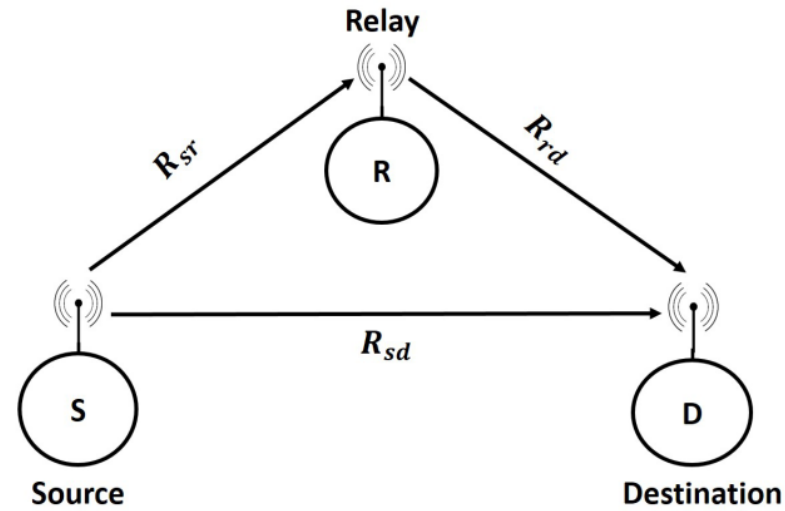

Fig. 1 Cooperative single-relay communication system model.

\section{SYSTEM MODEL}

We consider a half-duplex cooperative communication system model with a single-relay as shown in Fig. 1. Each terminal is not able to simultaneously transmit and receive data at the same time. The network is composed of a source terminal (S), a single relay terminal $(\mathrm{R})$ and a destination terminal (D). In Phase I, the source terminal transmits data signal directly to the destination terminal and the relay terminal at the same time. The received data signals $Y_{\mathrm{sd}}$ and $\mathrm{Y}_{\mathrm{sr}}$ at the intended destination terminal and the relay terminal, respectively, are given by

$$
\begin{aligned}
& Y_{s d}=R_{s d} X_{s}+a_{s d} \\
& Y_{s r}=R_{s r} X_{s}+a_{s r}
\end{aligned}
$$

where $R_{s d}$ is the gain coefficient of the S-D channel link, $X_{s}$ is the transmitted data signal, $a_{s d}$ is the noise added over the $\mathrm{S}-\mathrm{D}$ link, $R_{s r}$ is gain coefficient of S-R channel link and $a_{s r}$ is the noise added over the S-R link. In phase II, under the AF cooperative relaying technique, the relay terminal simply amplifies the incoming data signal then re-transmits it to intended destination terminal. The incoming data signal to the intended destination terminal from the relay terminal will be

$$
Y_{r d}=\alpha R_{r d} X_{s}+a_{r d}
$$

where $\alpha$ is amplification relaying factor, $R_{r d}$ is the gain coefficient of the R-D channel link and $a_{r d}$ is the noise added over the S-R link. Amplification relaying factor in the AF technique can be calculated as follows

$$
\alpha=\sqrt{\frac{\Psi}{|R|^{2} \Psi+2 \phi_{s r}^{2}}}
$$

where $\Psi=E\left[\left|X_{S}^{2}\right|\right]$ is the energy of transmitted data, $2 \phi_{s r}^{2}=E\left[\left|a_{s r}^{2}\right|\right]$ is the additive noise variance.

Under the SDF cooperative relaying technique, the relay terminal receives the sent data signal from the source terminal and decodes it such that the additive noise is not amplified. The SDF relay terminal is able to detect whether the received data contains errors or not and it will only transmit the correct data. The incoming data signal to the intended destination terminal from the relay terminal in this case is given by

$$
Y_{r d}=R_{r d} X_{r}+a_{r d}
$$

where $X_{r}$ is the transmitted data signal by the relay terminal.

Under the proposed EF cooperative relaying technique, the relay terminal receives the sent data signal from the source terminal at the first phase, then equalizes the S-R channel effect in the incoming data signal and finally forwards it to the intended destination terminal at the second phase. The incoming data signal to the intended destination terminal from the relay terminal in this case is given by

$$
Y_{r d}=R_{r d} X_{e}+a_{r d}
$$

where $X_{e}$ is the transmitted data signal by relay terminal. For any used cooperative relaying technique, the received data signals for each phase are combined using Equal ratio Combining (ERC) technique [7], in which the incoming data signals are equally weighted in the combination at the destination terminal. In this case the combination will be given by

$$
Y_{d}=\frac{1}{2}\left(Y_{s d}+Y_{r d}\right)
$$

\section{PROPOSED COOPERATIVE COMMUNICATION FRAMEWORK}

In this section, details of the experimental implementation of the proposed SDR-based cooperative communication system is discussed which is shown in Fig. 2. Software defined radio (SDR) is a fast rising technology in communication systems. The main SDR system comprises of anopen software GNU Radio running on a processor which implements baseband processing. National Instruments universal software radio peripheral (NI USRP) is used as an $\mathrm{RF}$ front end. It performs the up-conversion/downconversion and transmission/reception processes. ODROID XU4 lcite\{odroid\} serves as a processing unit for the relay terminal which enables portable standalone SDR framework implementation. ODROID XU4 offers many peripherals, such as USB 3.0 and Gigabit Ethernet interfaces, which is suitable for connection with many categories of NI USRPs whose bus connectors are Ethernet or USB 2 or USB 3. Also it has eMMC 5.0 which enables fast data transfer rate Moreover, it has an octa-core processor which enables efficient and powerful processing.

In order to carry out practical experiments with an RF device, the implementation needs to have the ability to cater for cases as carrier or timing offsets between the 
transmitter terminal and the receiver terminal. Therefore, packetized communication with a single carrier BPSK/QPSK transceiver is implemented. The BPSK/QPSK transmitter is designed as follows: First, 32 bits-CRC is added before data which is used as error detection for data. The data with CRC is modulated with BPSK/QPSK. Then, 32-bit header is created via the Packet Header Generator block. The header includes 12-bit data length, 12-bit packet number, and 8-bit $\mathrm{CRC}$ check on header to assure it is properly received. The header is modulated with BPSK. Finally, modulated header is appended before modulated data. The complex symbols are

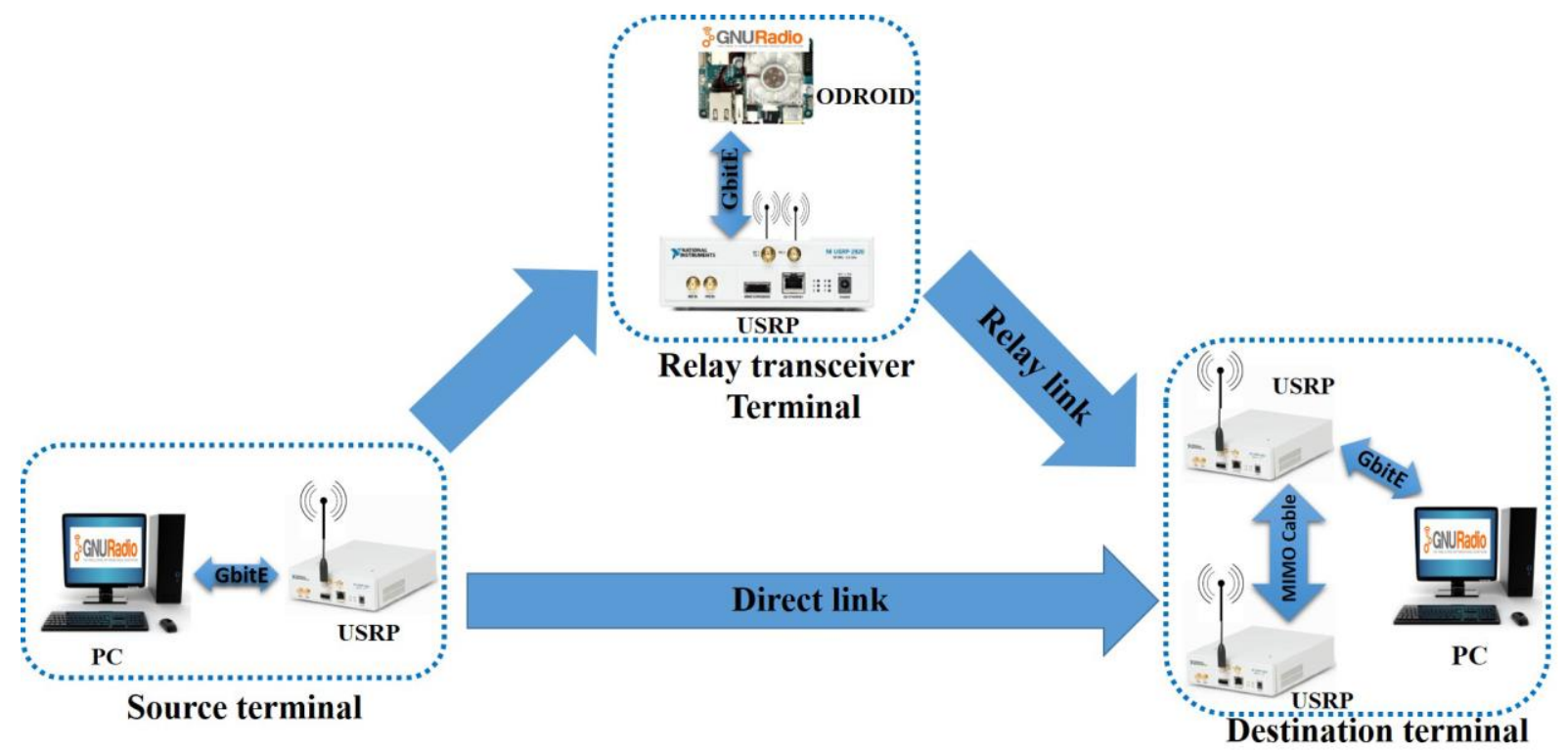

Fig. 2 Cooperative single-relay communication system implementation.

then passed to root raised cosine (RRC) filter for up sampling and pulse shaping to band-limit the transmission and minimize inter-symbol interference (ISI) effect at destination terminal.

The BPSK/QPSK receiver is designed as follows: First the receiving USRP down converts the carrier and prepares the IQ baseband symbols to host for further processing. The baseband signal is affected with fading, thermal noise, timing, phase and frequency offsets and ISI. To compensate these effects, BPSK/QPSK receiver is used. An automatic gain control (AGC) is used to keep a fixed gain of the received data to guarantee accurate operation of next synchronization operations. In order to detect a correlation peak, the incoming signal is correlated with the modulated reference preamble. Then to compensate for coarse frequency offset, frequency lock loop (FLL) is used. It locks on to carrier frequency and removes any carrier frequency offsets using a band edge filter. Then, the output from FLL is passed to a timing recovery module called polyphase Clock Sync which utilizes polyphase filter bank as explained in [8]. This module attains clock recovery. It also guarantees proper sampling of baseband signal and performs as a receiver matched filter. Then output is passed to Header/Payload Demux block to split the input stream into a header and data stream. In order to know the data length, it uses the feedback loop with the decoded header.

Then, for each stream a Constant Modulus Algorithm (CMA) equalizer is used which utilizes correction of multipath distortion. Finally, a Costas loop module is used to compensate for phase offsets. The next stage in receiving operation is decoding step where Costas loop is followed with a constellation demodulator which makes hard decision for received BPSK/QPSK symbols then maps these symbols to their equivalent bits. Protocol Parser block is used in header stream which extracts the header information from this packet so the subsequent data length will be passed to next step with a tag indicating packet number and data with CRC length.

\section{A. Source terminal}

At the source terminal shown in Fig 3, the data sequence is a text message. Then the resulting sequence is passed to the BPSK/QPSK transmitter that is described before. The matched-filtered and up-sampled baseband symbols are transmitted to the USRP Sink block which is used to connect the GNURadio flowgraph of source terminal with USRP hardware device. It up converts the incoming complex samples to be sent by the USRP device.

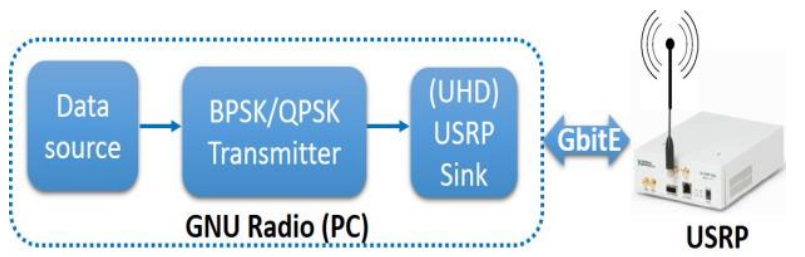

Fig 3 Source terminal architecture.

\section{B. Relay terminal}

The AF relay just amplifies and re-transmits the data signal to the destination terminal. The AF relay setup is shown in Fig. 4. The AF relay first receives the signal from the source terminal then performs fixed gain relaying (blind 
relaying) Icite\{li2010cooperative $\}$ which uses a fixed amplification gain of value 1 . Finally the relay terminal sends the amplified data to the destination terminal.

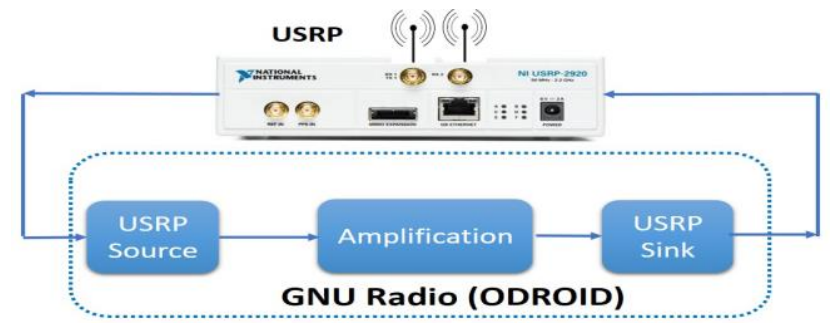

Fig. 4 AF relay terminal architecture

The DF relay can first demodulate and decode the incoming data signal, then re-encodes and re-modulates it. Finally, it sends the data to the intended destination terminal. The main advantage of the DF relaying technique is that by utilizing decoding process, the relay is able to basically exclude the noise accompanied by the re-sent signal. The implemented DF relay in this experiment forwards incoming data it receives from the source terminal utilizing a SDF relaying technique. A data sending decision is taken depending on an appended Cyclic Redundancy Check (CRC). So it differs from a normal DF relaying technique which sends any data that it receives so it repeals any diversity gain. As a result the Fixed DF technique is not analyzed here and all the practical results are assembled for the SDF technique. Experimental implementation for the SDF relay terminal is shown in Fig. 5.

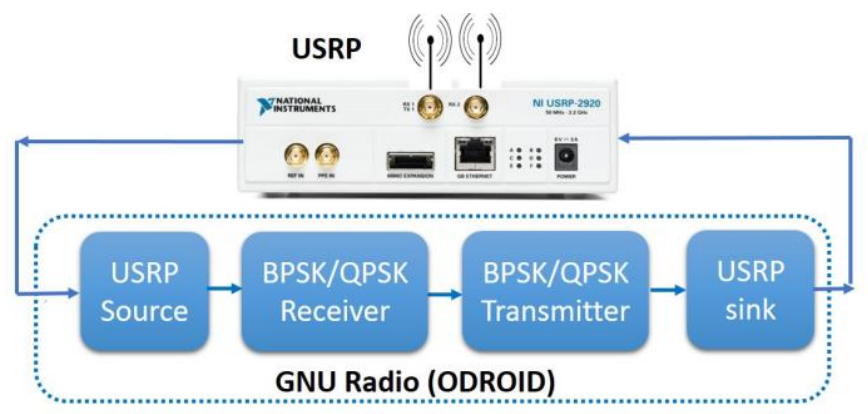

Fig. 5 SDF relay terminal architecture.

The EF relaying technique is simpler and less computational than the SDF relaying technique but some considerations have to be taken, because header received by the EF relay terminal can't be used once more. The EF relay terminal contains header generation phase with performing the equalization in the data flow at the same time. So, the destination terminal receives the equalized data with a new header generated in the EF relay terminal as shown in Fig. 6. The EF relaying technique performed is selective because Header/Payload Demux block require a trigger which allows the data recovery only if the verification of header is passed.

\section{Destination terminal}

The destination terminal is composed of two receiving NI USRPs which are aligned and synchronized with MIMO cable to receive data on two channels (channel one for receiving from source and channel two for relay). A receiving USRP is connected to PC through single Gigabit Ethernet and is connected to the other USRP through MIMO cable. The USRP device linked to Gigabit Ethernet functions as a switch and transport data from/to both USRP devices. It will handle sampling clock and time synchronization of data. First the receiving USRP carries out carrier down conversion and prepares IQ baseband symbols to host for further processing. Then, symbols are passed to BPSK/QPSK receiver except decoding process for data flow to recover the packet numbers for each detected packet. The signals corresponding to the same packet received are combined. Under the ERC technique, the ERC destination shown in Fig. 7 where both signals are equally weighted in the combination.

After the combination process, the combined symbols are passed to the BPSK/QPSK symbol de-mapping which makes hard symbol decision to recover the binary bits. Finally, the incoming bit stream is passed to data Sink block and is used to save the incoming stream out to a binary file to be used for further analysis.

\section{EXPERIMENTAL RESULTS}

The experiment is conducted on three-terminal network in lab environment. Table 1 shows the system parameters that are used during the experiments.

Table 1 Cooperative Relaying Implementation Parameters

\begin{tabular}{|c|c|}
\hline Parameters Value & Parameters Value \\
\hline Modulation BPSK/QPSK & Modulation BPSK/QPSK \\
\hline Operating Center Frequency & Operating Center Frequency \\
\hline 500 MHz for Direct Path, & $500 \mathrm{MHz}$ for Direct Path, \\
\hline 600 MHz for Relay Path. & $600 \mathrm{MHz}$ for Relay Path. \\
\hline Data Kind Text & Data Kind Text \\
\hline Bit Rate 250 Kbps & Bit Rate 250 Kbps \\
\hline Distance (S-D) 8 meters & Distance (S-D) 8 meters \\
\hline $\begin{array}{c}\text { Distance (S-R and R-D paths) } 4 \\
\text { meters }\end{array}$ & $\begin{array}{c}\text { Distance (S-R and R-D paths) } 4 \\
\text { meters }\end{array}$ \\
\hline
\end{tabular}

All the terminals are placed at identical distances which are about 4 meters from each other. The terminals setup is kept fixed through the overall experiment. The SNR vs. BER experimental curve is investigated. For performance analysis, 1000 packets are transmitted from the source terminal for each experiment. After applying AF or SDF or EF relaying techniques, and ERC techniques at the intended destination terminal, the averaged BER for 1000 packets are obtained.

Fig. 8 and Fig. 9 show the plot of experimental BER as a function of the estimated direct path SNR. It shows the BER performance comparison for direct transmission, AF, EF and SDF relaying techniques with ERC using BPSK and QPSK modulation schemes. The proposed EF relaying technique outperforms direct transmission and AF relaying technique. It also achieves comparable performance to the SDF relaying technique. 


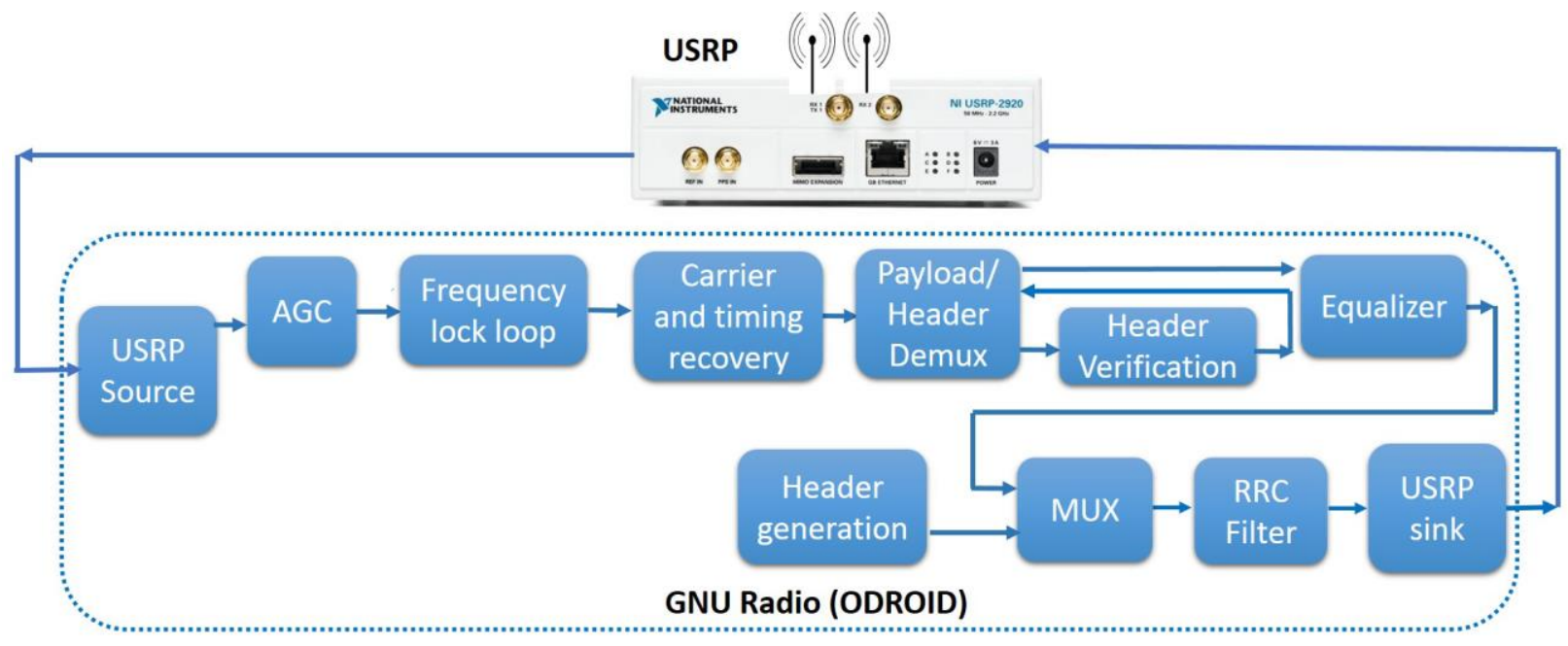

Fig. 6 EF relay terminal architecture.

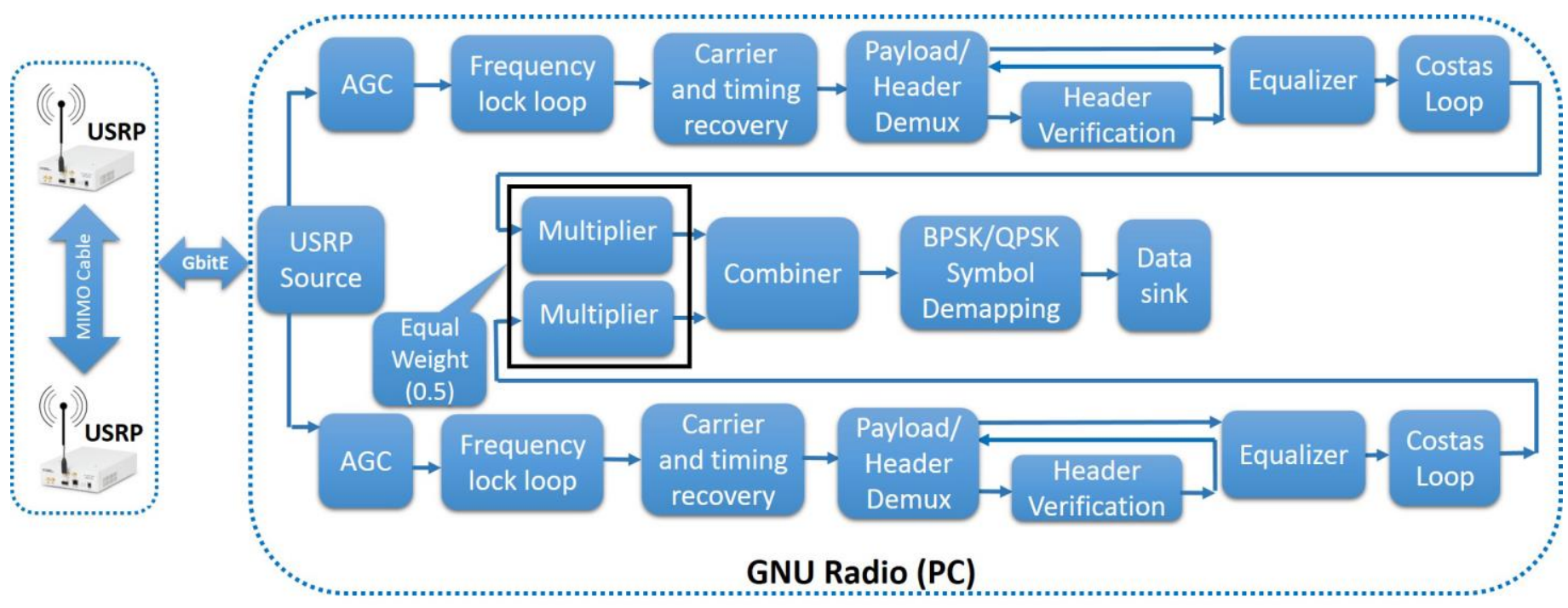

Fig. 7 ERC dExperimental BER for Direct transmission, AF, EF and SDF relaying using QPSK modulation. terminal architecture.

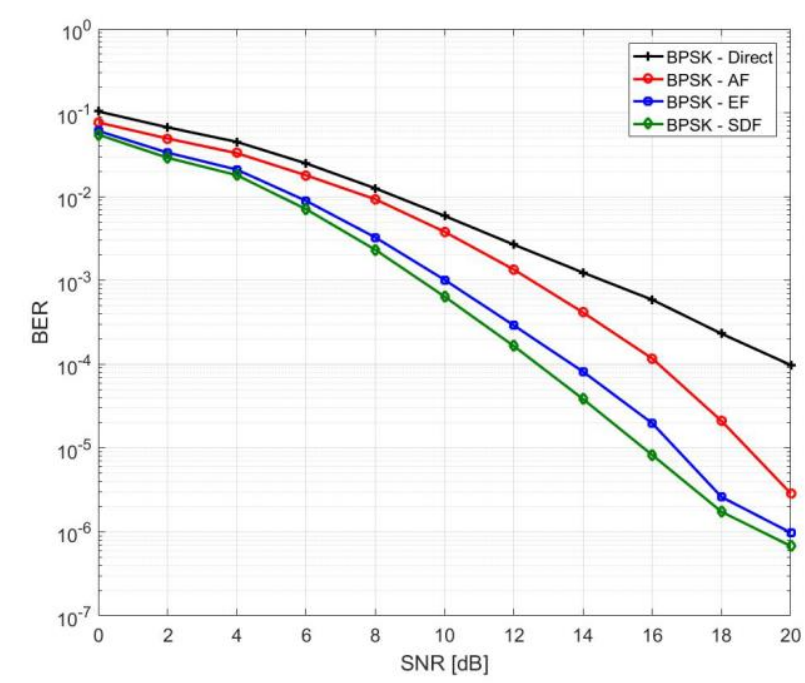

Fig. 8 Experimental BER for Direct transmission, AF, EF and SDF relaying using BPSK modulation.

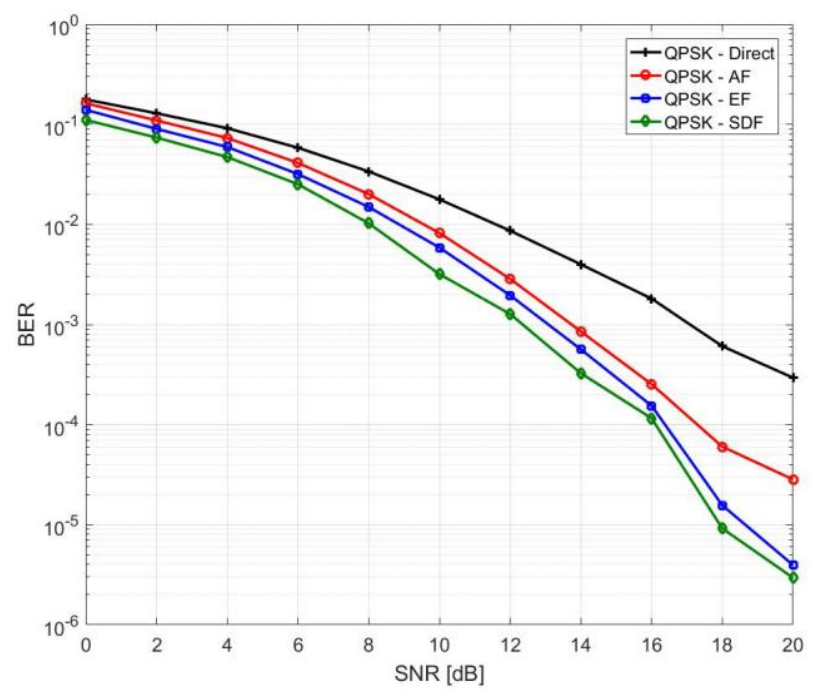

Fig. 9 Experimental BER for Direct transmission, AF, EF and SDF relaying using QPSK modulation. 


\section{CONCLUSION}

An efficient SDR based standalone cooperative communication system is presented. An EF relaying technique is proposed where the relay terminal equalizes the source-relay channel to mitigate the accumulation of the channel without the need of high computational processing and data regeneration. Experimental performance analysis shows that performance of cooperative communication outperforms direct communication. The proposed EF gives better performance than AF. Also, it gives comparable performance as the SDF relaying technique. In the future, expanding the implemented cooperative communication framework using multi-relays networks is planned. It can be expanded to future $5 \mathrm{G}$ wireless networks like Internet of Things and machine to machine communication.

\section{REFERENCES}

1. Bradford, G.J. and J.N. Laneman. An experimental framework for the evaluation of cooperative diversity. in 2009 43rd Annual Conference on Information Sciences and Systems. 2009. IEEE.

2. www.ettus.com, Universal Software Radio Peripheral. 2018.

3. www.gnuradio.org, GNU Radio. 2017.

4. Omar, M.S., et al. Experimental implementation of cooperative transmission range extension in indoor environments. in 2015 International Wireless Communications and Mobile Computing Conference (IWCMC). 2015. IEEE.

5. Prince, A., et al. Performance evaluation of multihop decode and forward cooperative relaying. in 2017 Intl Conf on Advanced Control Circuits Systems (ACCS) Systems \& 2017 Intl Conf on New Paradigms in Electronics \& Information Technology (PEIT). 2017. IEEE.

6. Tanoli, S., et al., An Experimental Channel Capacity Analysis of Cooperative Networks Using Universal Software Radio Peripheral (USRP). Sustainability, 2018. 10(6): p. 1983.

7. Akuon, P.O. and H. Xu, Optimal error analysis of receive diversity schemes on arbitrarily correlated Rayleigh fading channels. IET Communications, 2016. 10(7): p. 854-861.

8. Harris, F.J., Multirate signal processing for communication systems. 2004: Prentice Hall PTR. 